\title{
Convergence Theorems for $k$-Strictly Pseudononspreading Multivalued in Hilbert Spaces
}

\author{
Hongbo Liu*, Yi Li
}

School of Science, Southwest University of Science and Technology, Mianyang, China

Email: liuhongbo@swust.edu.cn

Received 15 May 2014; revised 15 June 2014; accepted 30 June 2014

Copyright (C) 2014 by authors and Scientific Research Publishing Inc.

This work is licensed under the Creative Commons Attribution International License (CC BY).

http://creativecommons.org/licenses/by/4.0/

(c) (i) Open Access

\section{Abstract}

We introduce a $k$-strictly pseudononspreading multivalued in Hilbert spaces more general than the class of nonspreading multivalued. We establish some weak convergence theorems of the sequences generated by our iterative process. Some new iterative sequences for finding a common element of the set of solutions for equilibrium problem was introduced. The results improve and extend the corresponding results of Osilike Isiogugu [1] (Nonlinear Anal.74 (2011)) and others.

\section{Keywords}

\section{Equilibrium Problem, $K$-Strictly Pseudononspreading Multivalued Mapping, Common Fixed Point}

\section{Introduction}

Throughout this paper, we denote by $\mathbb{N}$ and $\mathbb{R}$ the sets of positive integers and real numbers, respectively. Let $C$ be a nonempty closed subset of a real Hilbert space $H$. Let $N(C)$ and $C B(C)$ denote the family of nonempty subsets and nonempty closed bounded subsets of $E$, respectively. The Hausdorff metric on $C B(E)$ is defined by

$$
H\left(A_{1}, A_{2}\right):=\max \left\{\sup _{x \in A_{1}} d\left(x, A_{2}\right), \sup _{y \in A_{2}} d\left(y, A_{1}\right)\right\}
$$

for $A_{1}, A_{2} \in C B(C)$, where $d\left(x, A_{1}\right)=\inf \left\{\|x-y\|, y \in A_{1}\right\}$. An element $p \in E$ is called a fixed point of a multivalued mapping $T: C \rightarrow N(C)$ if $p \in T(p)$. The set of fixed points of a multivalued mapping $T$ is

${ }^{*}$ Corresponding author. 
represented by $F(T)$.

The multivalued mapping $T: C \rightarrow C B(C)$ is called nonexpansive if

$$
H(T x, T y) \leq\|x-y\|, \quad \forall x, y \in C .
$$

The multivalued mapping $T: C \rightarrow C B(C)$ is called quasi-nonexpansive if $F(T) \neq \varnothing$ and

$$
H(T x, T p) \leq\|x-p\|, \quad \text { for } \forall x \in C \quad p \in F(T) .
$$

Iterative process for approximating fixed points (and common fixed points) of nonexpansive multivalued mappings have been investigated by various authors (see [2]-[5]).

Recently, Kohsaka and Takahashi (see [6] [7]) introduced an important class of mappings which they called the class of nonspreading mappings. Let $C$ be a subset of Hilbert space $H$, they called a mapping $T: C \rightarrow C$ nonspreading if

$$
2\|T x-T y\|^{2} \leq\|T x-y\|^{2}+\|T y-x\|^{2} \quad \forall x, y \in C .
$$

Lemoto and Takahashi [8] proved that $T: C \rightarrow C$ is nonspreading if and only if

$$
\|T x-T y\|^{2} \leq\|x-y\|^{2}+2\langle x-T x, y-T y\rangle \quad \forall x, y \in C .
$$

Now, inspired by [6] and [7], we propose a definition as follows.

Definition 1.1 The multivalued mapping $T: C \rightarrow C B(C)$ is called nonspreading if

$$
2\left\|u_{x}-u_{y}\right\|^{2} \leq\left\|u_{x}-y\right\|^{2}+\left\|u_{y}-x\right\|^{2} \quad \text { for } u_{x} \in T x, u_{y} \in T y, \forall x, y \in C .
$$

By Takahashi [8], We get also the multivalued mapping $T: C \rightarrow C B(C)$ is nonspreading if and only if

$$
\left\|u_{x}-u_{y}\right\|^{2} \leq\|x-y\|^{2}+2\left\langle x-u_{x}, y-u_{y}\right\rangle \text { for } u_{x} \in T x, u_{y} \in T y, \forall x, y \in C .
$$

Infact,

$$
\begin{aligned}
& 2\left\|u_{x}-u_{y}\right\|^{2} \leq\left\|u_{x}-y\right\|^{2}+\left\|u_{y}-x\right\|^{2} \\
& \Leftrightarrow 2\left\|u_{x}-u_{y}\right\|^{2} \leq\left\|u_{x}-x\right\|^{2}+2\left\langle u_{x}-x, x-y\right\rangle+\|x-y\|^{2} \\
& +\left\|u_{x}-x\right\|^{2}+2\left\langle x-u_{x}, u_{x}-u_{y}\right\rangle+\left\|u_{x}-u_{y}\right\|^{2} \\
& \Leftrightarrow 2\left\|u_{x}-u_{y}\right\|^{2} \leq 2\left\|u_{x}-x\right\|^{2}+\|x-y\|^{2}+\left\|u_{x}-u_{y}\right\|^{2}+2\left\langle u_{x}-x, x-u_{x}-\left(y-u_{y}\right)\right\rangle \\
& \Leftrightarrow\left\|u_{x}-u_{y}\right\|^{2} \leq\|x-y\|^{2}+2\left\langle x-u_{x}, y-u_{y}\right\rangle
\end{aligned}
$$

Definition 1.2 The multivalued mapping $T: C \rightarrow C B(C)$ is called $k$-strictly pseudononspreading if there exists $k \in[0,1)$ such that

$$
\left\|u_{x}-u_{y}\right\|^{2} \leq\|x-y\|^{2}+k\left\|x-u_{x}-\left(y-u_{y}\right)\right\|^{2}+2\left\langle x-u_{x}, y-u_{y}\right\rangle \text {, for } u_{x} \in T x, u_{y} \in T y, \forall x, y \in C .
$$

Observe that suppose $T$ is k-strictly pseudononspreading with $F(T) \neq \varnothing$, and $p \in F(T) \Rightarrow T p=\{p\}$, then

$$
\left\|u_{x}-p\right\|^{2} \leq\|x-p\|^{2}+k\left\|x-u_{x}\right\|^{2} \quad x \in C, u_{x} \in T x, p \in F(T) .
$$

Clearly every nonspreading multivalued mapping is $k$-strictly pseudononspreading multivalued mapping. The following example shows that the class of $k$-strictly pseudononspreading mappings is more general than the class of nonspreading mappings.

Example (see [1] page 1816 Example 1), Let $\mathscr{h}$ denote the reals with the usual norm. Let $T: \mathscr{h} \rightarrow \mathscr{h}$ be defined for each $x \in \mathscr{R}$ by

$$
T x= \begin{cases}x, & x \in(-\infty, 0) \\ -2 x, & x \in[0, \infty)\end{cases}
$$


The equilibrium problem for $\phi: E \times E \rightarrow R$ is to find $x \in E$ such that $\phi(x, y) \geq 0, \forall y \in E$. The set of such solution is denoted by $E P(\phi)$. Given a mapping $T: E \rightarrow C B(E)$, let $\phi(x, y)=\langle x, y\rangle$ for all $y \in E$. The $x \in E P(\phi)$ if and only if $x \in E$ is a solution of the variational inequality $\langle T x, y\rangle \geq 0$ for all $y \in E$.

Numerous problems in physics, optimization, and economics can be reduced to find a solution of the equilibrium problem. Some methods have been proposed to solve the equilibrium problem see, for instance, Blum and Oettli [9], Combettes and Hirstoaga [10], Li and Li [11], Giannessi, Maugeri, and Pardalos [12], Moudafi and Thera [13] and Pardalos, Rassias and Khan [14], Ceng et al. [15]. In the recent years, the problem of finding a common element of the set of solutions of equilibrium problems and the set of fixed points of single-valued nonexpansive mappings in the framework of Hilbert spaces has been intensively studied by many authors.

In this paper, inspired by [1] we propose an iterative process for finding a common element of the set of solutions of equilibrium problem and the set of common fixed points of k-strictly pseudononspreading multivalued mapping in the setting of real Hilbert spaces. We also prove the strong and weak convergence of the sequences generated by our iterative process. The results presented in the paper improve and extend the corresponding results in [1] and others.

\section{Preliminaries and Lemma}

In the sequel, we begin by recalling some preliminaries and lemmas which will be used in the proof.

Lemma 2.1 Let $H$ be a real Hilbert space, for all $x, y \in H$ and $t \in[0,1]$, then the following well known results hold:

(i) $\|x+y\|^{2} \leq\|x\|^{2}+2\langle x+y, y\rangle$.

(ii) $\|t x+(1-t) y\|^{2}=t\|x\|^{2}+(1-t)\|y\|^{2}-t(1-t)\|x-y\|^{2}$

(iii) If $\left\{x_{n}\right\}$ is a sequence in $H$ which converges weakly to $z \in H$ then

$$
\limsup _{n \rightarrow \infty}\left\|x_{n}-y\right\|^{2}=\limsup _{n \rightarrow \infty}\left\|x_{n}-z\right\|^{2}+\|z-y\|^{2}
$$

Let $C$ be a nonempty closed convex subset of a real Hilbert space $H$. The nearest point projection $P_{C}: H \rightarrow C$ defined from $H$ onto $C$ is the function which assigns to each $x \in H$ its nearest point denoted by $P_{C} x$ in $C$. Thus $P_{C} x$ is the unique point in $C$ such that

$$
\left\|x-P_{C} x\right\| \leq\|x-y\|, \quad \forall y \in C .
$$

It is known that for each $x \in H$

$$
\left\langle x-P_{C} x, y-P_{C} x\right\rangle \leq 0, \quad \forall y \in C .
$$

Lemma 2.2 (see [5]) Let $C$ be a nonempty closed convex subset of a real Hilbert space $H$. Let $P_{C}: H \rightarrow C$ be the metric projection of $H$ onto $C$. Let $\left\{x_{n}\right\}$ be a sequence in $C$ and let $\left\|x_{n+1}-u\right\| \leq\left\|x_{n}-u\right\|$ for all $u \in C$. Then $\left\{P_{C} X_{n}\right\}$ converges strongly.

We present the following properties of a k-strictly pseudononspreading multivalued mapping.

Lemma 2.3 Let $C$ be a nonempty closed convex subset of a real Hilbert space $H$, and let $T: C \rightarrow C B(C)$ be a k-strictly pseudononspreading multivalued mapping. If $F(T) \neq \varnothing$, and $p \in F(T) \Rightarrow T p=\{p\}$, then it is closed and convex.

Proof. Let $\left\{x_{n}\right\} \subseteq F(T)$ and $x_{n} \rightarrow x \in C$ (as $n \rightarrow \infty$ ). Since $\left\|u_{x}-x\right\| \leq\left\|u_{x}-u_{x_{n}}\right\|+\left\|x_{n}-x\right\|$ and

$$
\left\|u_{x}-u_{x_{n}}\right\|^{2} \leq\left\|x_{n}-x\right\|^{2}+k\left\|u_{x}-x\right\|^{2} \leq\left(\left\|x_{n}-x\right\|+\sqrt{k}\left\|u_{x}-x\right\|\right)^{2} \text {, }
$$

we have $0 \leq\left\|u_{x}-x\right\| \leq \frac{2}{1-\sqrt{k}}\left\|x_{n}-x\right\| \rightarrow 0 \quad$ (as $n \rightarrow \infty$ ). Hence $x \in F(T)$.

Next let $z=t p+(1-t) q$, where $p, q \in F(T)$ and $0 \leq t \leq 1$, we have

$$
\begin{aligned}
& \left\|z-u_{z}\right\|^{2}=\left\|t\left(p-u_{z}\right)+(1-t)\left(q-u_{z}\right)\right\|^{2}=t\left\|\left(p-u_{z}\right)\right\|^{2}+(1-t)\left\|q-u_{z}\right\|^{2}-t(1-t)\|p-q\|^{2} \\
& \leq t\left[\|p-z\|^{2}+k\left\|z-u_{z}\right\|^{2}\right]+(1-t)\left[\|q-z\|^{2}+k\left\|z-u_{z}\right\|^{2}\right]-t(1-t)\|p-q\|^{2}=k\left\|z-u_{z}\right\|^{2}, \quad u_{z} \in T z,
\end{aligned}
$$


Thus $\left\|z-u_{z}\right\|=0$ and hence $z \in F(T)$. This complete the proof of Lemma 2.3

Lemma 2.4 Let $C$ be a nonempty closed convex subset of a real Hilbert space $H$, and let $T: C \rightarrow C B(C)$ be a $k$-strictly pseudononspreading multivalued mapping. If $F(T) \neq \varnothing$, and $p \in F(T) \Rightarrow T p=\{p\}$, then $I-T$ is demiclosed at 0 .

Proof. Let $\left\{x_{n}\right\}$ be a sequence in $C$ which $x_{n} \rightarrow p$ and $x_{n}-u_{x_{n}} \rightarrow 0$ (as $n \rightarrow \infty$ ).

Since $x_{n} \rightarrow p$, it is bounded. For each $x \in H$ define $\phi: H \rightarrow[0,+\infty)$ by

$$
\phi(x)=\limsup _{n \rightarrow \infty}\left\|x_{n}-x\right\|^{2} .
$$

Then from Lemma 2.1 we obtain

$$
\phi(x)=\limsup _{n \rightarrow \infty}\left\|x_{n}-p\right\|^{2}+\|p-x\|^{2}=\phi(p)+\|p-x\|^{2} \quad \forall x \in H,
$$

and so $\phi\left(u_{p}\right)=\phi(p)+\left\|p-u_{p}\right\|^{2}$ (where $u_{p} \in T p$ ).

In addition,

$$
\begin{aligned}
\phi\left(u_{p}\right) & =\limsup _{n \rightarrow \infty}\left\|x_{n}-u_{p}\right\|^{2}=\underset{n \rightarrow \infty}{\limsup }\left\|u_{x_{n}}-u_{p}\right\|^{2} \\
& \leq \limsup _{n \rightarrow \infty}\left\|x_{n}-p\right\|^{2}+k\left\|p-u_{p}\right\|^{2} \\
& =\phi(p)+k\left\|p-u_{p}\right\|^{2} .
\end{aligned}
$$

We obtain $(1-k)\left\|p-u_{p}\right\|^{2}=0$. Thus $\left\|p-u_{p}\right\|=0$ and hence $p \in F(T)$. This complete the proof of Lemma 2.4.

\section{Main Results}

Theorem 3.1 Let $C$ be a nonempty closed convex subset of a real Hilbert space $H$, and let $T: C \rightarrow C B(C)$ be a k-strictly pseudononspreading multivalued mapping with $F(T) \neq \varnothing$ and $p \in F(T) \Rightarrow T p=\{p\}$. Let $\beta \in[k, 1)$ and $\left\{\alpha_{n}\right\}$ be a real sequence in $[0,1)$ such that $\lim _{n \rightarrow \infty} \alpha_{n}=0$. Let $\left\{x_{n}\right\}$ and $\left\{z_{n}\right\}$ be sequences generated initially by an arbitrary element $x_{1} \in C$ and then by

$$
\left\{\begin{array}{l}
x_{n+1}=\alpha_{n} x_{n}+\left(1-\alpha_{n}\right) y_{n} \\
y_{n}=\beta x_{n}+(1-\beta) u_{x_{n}} \quad u_{x_{n}} \in T x_{n}, n \geq 1 \\
z_{n}=\frac{1}{n} \sum_{i=1}^{n} x_{i} \quad n \geq 1
\end{array}\right.
$$

Then, the sequences $\left\{z_{n}\right\}$ converge weakly to $z \in F(T)$, where $z=\lim _{n \rightarrow \infty} P_{F(T)} X_{n}$.

Proof. Let $T^{\beta}:=\beta I+(1-\beta) T$

First, We claim that $F\left(T^{\beta}\right)=F(T)$.

Indeed, if $p \in F(T)$, then

$$
u_{p}^{\beta}=(\beta I+(1-\beta) T) p=\beta p-(1-\beta) u_{p}=p, \quad u_{p}^{\beta} \in T^{\beta} p,
$$

this implies $F\left(T^{\beta}\right)=F(T)$ and $p \in T^{\beta} p \Rightarrow T^{\beta} p=\{p\}$ Next, for $\forall x, y \in C$ we have

$$
\begin{aligned}
\left\|u_{x}^{\beta}-u_{y}^{\beta}\right\|^{2} & =\left\|\beta(x-y)+(1-\beta)\left(u_{x}-u_{y}\right)\right\|^{2} \\
& =\beta\|x-y\|^{2}+(1-\beta)\left\|u_{x}-u_{y}\right\|^{2}-\beta(1-\beta)\left\|\left(x-u_{x}\right)-\left(y-u_{y}\right)\right\|^{2} .
\end{aligned}
$$


By (1.3) and (3.1), we obtain

$$
\left\|u_{x}^{\beta}-u_{y}^{\beta}\right\|^{2} \leq\|x-y\|^{2}+\frac{2}{1-\beta}\left\langle x-u_{x}^{\beta}, y-u_{y}^{\beta}\right\rangle .
$$

Observe also that for each $p \in F(T)$

$$
\begin{aligned}
\left\|x_{n+1}-p\right\| & =\left\|\alpha_{n}\left(x_{n}-p\right)+\left(1-\alpha_{n}\right)\left(u_{x_{n}}^{\beta}-p\right)\right\| \\
& \leq \alpha_{n}\left\|x_{n}-p\right\|+\left(1-\alpha_{n}\right)\left\|u_{x_{n}}^{\beta}-p\right\| \leq\left\|x_{n}-p\right\|,
\end{aligned}
$$

hence $\left\{x_{n}\right\}$ is bounded. By Lemma 2.1 and (3.2), we obtain

$$
\begin{aligned}
\left\|x_{n+1}-u_{y}^{\beta}\right\|^{2} & =\left\|\alpha_{n}\left(x_{n}-u_{y}^{\beta}\right)+\left(1-\alpha_{n}\right)\left(u_{x_{n}}^{\beta}-u_{y}^{\beta}\right)\right\|^{2} \\
& =\alpha_{n}\left\|x_{n}-u_{y}^{\beta}\right\|^{2}+\left(1-\alpha_{n}\right)\left\|u_{x_{n}}^{\beta}-u_{y}^{\beta}\right\|^{2}-\alpha_{n}\left(1-\alpha_{n}\right)\left\|x_{n}-u_{x_{n}}^{\beta}\right\|^{2} \\
& \leq \alpha_{n}\left\|x_{n}-u_{y}^{\beta}\right\|^{2}+\left(1-\alpha_{n}\right)\left[\left\|x_{n}-y\right\|^{2}+\frac{2}{1-\beta}\left\langle x_{n}-u_{x_{n}}^{\beta}, y-u_{y}^{\beta}\right\rangle\right] .
\end{aligned}
$$

Since

$$
\left\|x_{n}-y\right\|^{2}=\left\|x_{n}-u_{y}^{\beta}\right\|^{2}+\left\|u_{y}^{\beta}-y\right\|^{2}+2\left\langle x_{n}-u_{y}^{\beta}, u_{y}^{\beta}-y\right\rangle,
$$

it follows from (3.3) and (3.4) that

$$
\begin{aligned}
\left\|x_{n+1}-u_{y}^{\beta}\right\|^{2} \leq & \left\|x_{n}-u_{y}^{\beta}\right\|^{2}+\left\|u_{y}^{\beta}-y\right\|^{2}+2\left\langle x_{n}-u_{y}^{\beta}, u_{y}^{\beta}-y\right\rangle \\
& -2 \alpha_{n}\left\langle x_{n}-u_{y}^{\beta}, u_{y}^{\beta}-y\right\rangle+\frac{2}{1-\beta}\left\langle x_{n}-x_{n+1}, u_{y}^{\beta}-y\right\rangle .
\end{aligned}
$$

Summing (3.5) from $n=1$ to $n$, and dividing by $n$ we obtain

$$
\begin{aligned}
\frac{1}{n}\left\|x_{n+1}-u_{y}^{\beta}\right\|^{2} \leq & \frac{1}{n}\left\|x_{1}-u_{y}^{\beta}\right\|^{2}+\frac{2}{1-\beta}\left\langle\frac{x_{1}}{n}-\frac{x_{n+1}}{n},-y+u_{y}^{\beta}\right\rangle+\left\|u_{y}^{\beta}-y\right\|^{2} \\
& +2\left\langle z_{n}-u_{y}^{\beta}, u_{y}^{\beta}-y\right\rangle-\frac{2}{n} \sum_{i=1}^{n} \alpha_{i}\left\langle x_{i}-u_{y}^{\beta}, u_{y}^{\beta}-y\right\rangle .
\end{aligned}
$$

Since $\left\{x_{n}\right\}$ is bounded,then $\left\{z_{n}\right\}$ is also bounded. Thus there exists a subsequence $\left\{z_{n_{j}}\right\}$ of $\left\{z_{n}\right\}$ such that $z_{n_{j}} \rightarrow w($ as $j \rightarrow \infty)$. we also have

$$
\begin{aligned}
\frac{1}{n_{j}}\left\|x_{n_{j}+1}-u_{y}^{\beta}\right\|^{2} \leq & \frac{1}{n_{j}}\left\|x_{1}-u_{y}^{\beta}\right\|^{2}+\frac{2}{1-\beta}\left\langle\frac{x_{1}}{n_{j}}-\frac{x_{n_{j}+1}}{n_{j}},-y+u_{y}^{\beta}\right\rangle+\left\|u_{y}^{\beta}-y\right\|^{2} \\
& +2\left\langle z_{n_{j}}-u_{y}^{\beta}, u_{y}^{\beta}-y\right\rangle-\frac{2}{n_{j}} \sum_{i=1}^{n_{j}} \alpha_{i}\left\langle x_{i}-u_{y}^{\beta}, u_{y}^{\beta}-y\right\rangle .
\end{aligned}
$$

As $j \rightarrow \infty$ we obtain from (3.7) that

$$
0 \leq\left\|u_{y}^{\beta}-y\right\|^{2}+2\left\langle w-u_{y}^{\beta}, u_{y}^{\beta}-y\right\rangle .
$$

Since $y \in H$ was arbitrary, setting $y=w$ in (3.8) we have

$$
0 \leq\left\|u_{w}^{\beta}-w\right\|^{2}-2\left\|u_{w}^{\beta}-w\right\|^{2},
$$

from which it follows that $w \in F\left(T^{\beta}\right)=F(T)$. Since $F(T)$ is closed and convex by Lemma 2.3, thus we can define the projection $P_{F(T)}$. 
From Lemma 2.2, $\left\{P_{F(T)} X_{n}\right\}$ converges strongly. Let $\lim _{n \rightarrow \infty} P_{F(T)} X_{n}=z$.

Next we show that $z=w$.

Since $\left\{x_{n}\right\}$ and $\left\{P_{F(T)} x_{n}\right\}$ are bounded, there exists $M>0$ such that $\left\|x_{n}-P_{F(T)} x_{n}\right\| \leq M \quad(\forall n \geq 1)$, then we obtain by $\left\langle w-P_{F(T)} X_{n}, x_{n}-P_{F(T)} x_{n}\right\rangle \leq 0$

$$
\begin{aligned}
\left\langle w-z, x_{n}-P_{F(T)} x_{n}\right\rangle & =\left\langle w-P_{F(T)} X_{n}, x_{n}-P_{F(T)} x_{n}\right\rangle+\left\langle P_{F(T)} x_{n}-z, x_{n}-P_{F(T)} X_{n}\right\rangle \\
& \leq\left\langle P_{F(T)} X_{n}-z, x_{n}-P_{F(T)} x_{n}\right\rangle \leq\left\|P_{F(T)} X_{n}-z\right\| \cdot\left\|x_{n}-P_{F(T)} X_{n}\right\| \\
& \leq M\left\|P_{F(T)} x_{n}-z\right\|
\end{aligned}
$$

Summing (3.9) from $n=1$ to $n_{j}$, and dividing by $n_{j}$ we obtain

$$
\left\langle w-z, z_{n_{j}}-\frac{1}{n_{j}} \sum_{i=1}^{n_{j}} P_{F(T)} X_{i}\right\rangle \leq \frac{M}{n_{j}} \sum_{i=1}^{n_{j}}\left\|P_{F(T)} X_{i}-z\right\| .
$$

Sine $z_{n_{j}} \rightarrow w$ as $j \rightarrow \infty$, and $\lim _{n \rightarrow \infty} P_{F(T)} X_{n}=z$, we have

$$
\langle w-z, w-z\rangle=\|w-z\|^{2} \leq 0 .
$$

Hence $w=z$, so, the sequences $\left\{z_{n}\right\}$ converge weakly to $z \in F(T)$, where $z=\lim _{n \rightarrow \infty} P_{F(T)} X_{n}$. This complete the proof of Theorem 3.1.

\section{Acknowledgments}

This work is supported by the Doctoral Program Research Foundation of Southwest University of Science and Technology (No.11zx7129) and the National Natural Science Foundation of China (No.71071102).

The authors are very grateful to the referees for their helpful comments and valuable suggestions.

\section{References}

[1] Osilike, M.O. and Isiogugu, F.O. (2011) Weak and Strong Convergence Theorems for Nonspreading-Type Mappings in Hilbert Spaces. Nonlinear Analysis: Theory, Methods \& Applications, 74, 1814-1822. http://dx.doi.org/10.1016/j.na.2010.10.054

[2] Song, Y. and Wang, H. (2009) Convergence of Iterative Algorithms for Multivalued Mappings in Banach Spaces. Nonlinear Analysis, 70, 1547-1556. http://dx.doi.org/10.1016/j.na.2008.02.034

[3] Shahzad, N. and Zegeye, H. (2009) On Mann and Ishikawa Iteration Schemes for Multivalued Maps in Banach Space. Nonlinear Analysis, 71, 838-844. http://dx.doi.org/10.1016/j.na.2008.10.112

[4] Eslamian, M. and Abkar, A. (2011) One-Step Iterative Process for a Finite Family of Multivalued Mappings. Mathematical and Computer Modelling, 54, 105-111. http://dx.doi.org/10.1016/j.mcm.2011.01.040

[5] Takahashi, W. and Toyoda, M. (2003) Weak Convergence Theorems for Nonexpansive Mappings and Monotone Mappings. Journal of Optimization Theory and Applications, 118, 417-428. http://dx.doi.org/10.1023/A:1025407607560

[6] Kohsaka, F. and Takahashi, W. (2008) Fixed Point Theorems for a Class of Nonlinear Mappings Relate to Maximal Monotone Operators in Banach Spaces. Archiv der Mathematik (Basel), 91, 166-177. http://dx.doi.org/10.1007/s00013-008-2545-8

[7] Kohsaka, F. and Takahashi, W. (2008) Existence and Approximation of Fixed Points of Firmly Nonexpansive-Type Mappings in Banach Spaces. SIAM Journal on Optimization, 19, 824-835. http://dx.doi.org/10.1137/070688717

[8] Iemoto, S. and Takahashi, W. (2009) Approximating Commom Fixed Points of Nonexpansive Mappings and Nonspreading Mappings in a Hilbert Space. Nonlinear Analysis, 71, 2082-2089. http://dx.doi.org/10.1016/j.na.2009.03.064

[9] Blum, E. and Oettli, W. (1994) From Optimization and Variational Inequalities to Equilibrium Problems. The Mathematics Student, 63, 123-145. 
[10] Combettes, P.L. and Hirstoaga, S.A. (2005) Equilibrium Programming in Hilbert Spaces. Journal of Nonlinear and Convex Analysis, 6, 117-136.

[11] Li, X.B. and Li, S.J. (2010) Existence of Solutions for Generalized Vector Quasi-Equilibrium Problems. Optimization Letters, 4, 17-28. http://dx.doi.org/10.1007/s11590-009-0142-9

[12] Giannessi, F., Maugeri, G. and Pardalos, P.M. (2001) Equilibrium Problems: Nonsmooth Optimization and Variational Inequality Models. Kluwer Academics Publishers, Dordrecht.

[13] Moudafi, A. and Thera, M. (1999) Proximal and Dynamical Approaches to Equilibrium Problems, In: Lecture Note in Economics and Mathematical Systems, Vol. 477, Springer-Verlag, New York, 187-201.

[14] Pardalos, P.M., Rassias, T.M. and Khan, A.A. (2010) Nonlinear Analysis and Variational Problems. Springer, Berlin. http://dx.doi.org/10.1007/978-1-4419-0158-3

[15] Ceng, L.C., Al-Homidan, S., Ansari, Q.H. and Yao, J.C. (2009) An Iterative Scheme for Equilibrium Problems and Fixed Point Problems of Strict Pseudo-Contraction Mappings. Journal of Computational and Applied Mathematics, 223, 967-974. http://dx.doi.org/10.1016/j.cam.2008.03.032 
Scientific Research Publishing (SCIRP) is one of the largest Open Access journal publishers. It is currently publishing more than 200 open access, online, peer-reviewed journals covering a wide range of academic disciplines. SCIRP serves the worldwide academic communities and contributes to the progress and application of science with its publication.

Other selected journals from SCIRP are listed as below. Submit your manuscript to us via either submit@scirp.org or Online Submission Portal.
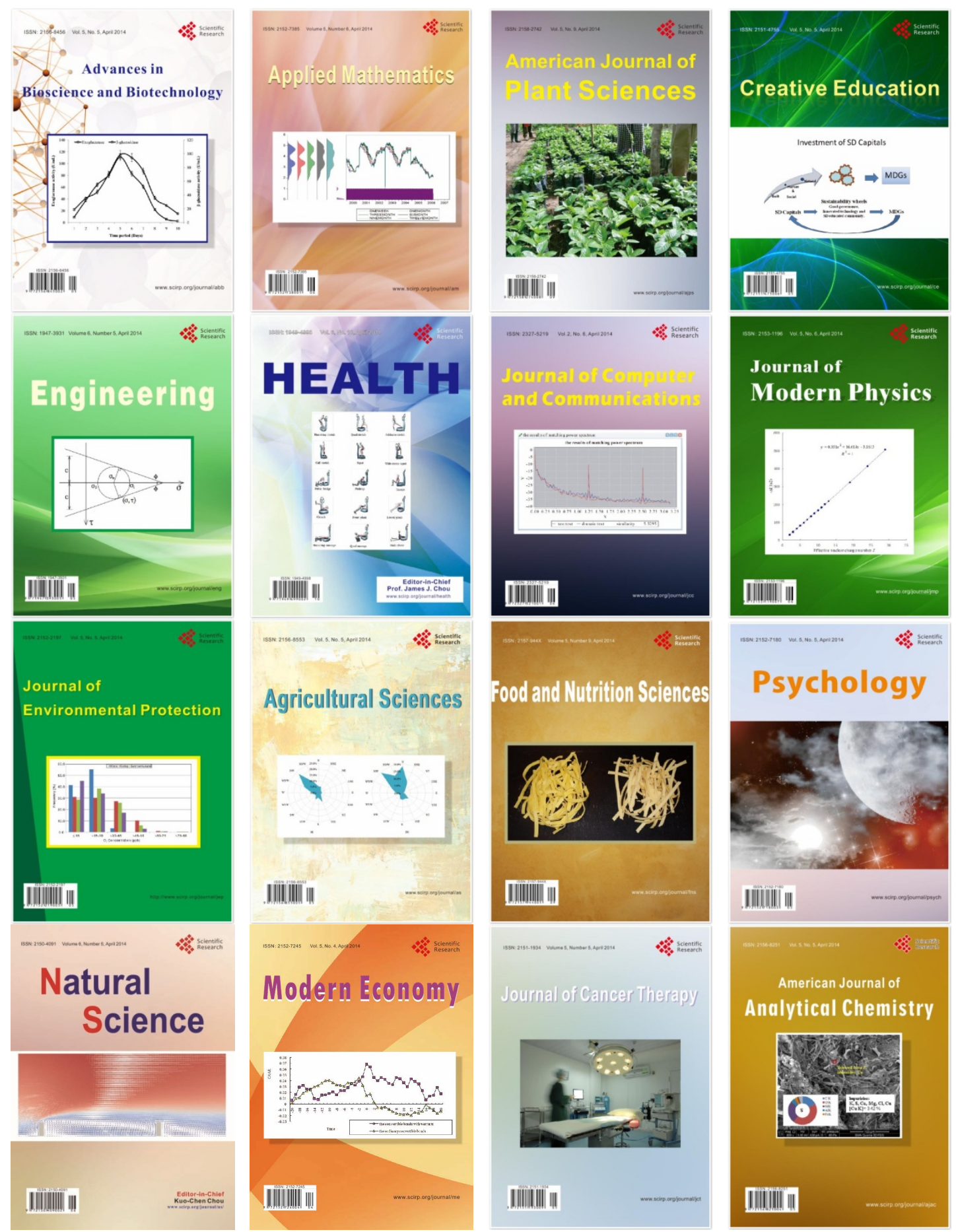